\title{
Nonlinear silicon photonics
}

\author{
Kevin K. Tsia*a,b ${ }^{* a h r a m ~ J a l a l i * a ~}$ \\ ${ }^{a}$ UCLA Photonics Laboratory, University of California, Los Angeles, CA, 90095 USA; \\ ${ }^{\mathrm{b}}$ Current address: Department of Electrical and Electronic Engineering, \\ The University of Hong Kong, Pokfulam Road, Hong Kong \\ Email:*tsia@hku.hk;**jalali@ucla.edu
}

\begin{abstract}
An intriguing optical property of silicon is that it exhibits a large third-order optical nonlinearity, with orders-ofmagnitude larger than that of silica glass in the telecommunication band. This allows efficient nonlinear optical interaction at relatively low power levels in a small footprint. Indeed, we have witnessed a stunning progress in harnessing the Raman and Kerr effects in silicon as the mechanisms for enabling chip-scale optical amplification, lasing, and wavelength conversion - functions that until recently were perceived to be beyond the reach of silicon. With all the continuous efforts developing novel techniques, nonlinear silicon photonics is expected to be able to reach even beyond the prior achievements. Instead of providing a comprehensive overview of this field, this manuscript highlights a number of new branches of nonlinear silicon photonics, which have not been fully recognized in the past. In particular, they are two-photon photovoltaic effect, mid-wave infrared (MWIR) silicon photonics, broadband Raman effects, inverse Raman scattering, and periodically-poled silicon (PePSi). These novel effects and techniques could create a new paradigm for silicon photonics and extend its utility beyond the traditionally anticipated applications.
\end{abstract}

Keywords: silicon photonics, nonlinear optics

\section{INTRODUCTION}

The burgeoning of silicon photonics over the past eight years has proven it to be now the most active discipline within the field of integrated optics [1-3]. The primary argument in favor of silicon photonics is based on its compatibility with the mighty silicon IC manufacturing. Another key motivation is to exploit silicon's optical nonlinear properties to build a myriad of chip-scale active silicon photonic devices. Such effort is driven by the fact that the large index contrast between the silicon and silicon dioxide - a gift offered by the silicon-on-insulator (SOI) platform, results in the high optical nonlinearity in SOI waveguides. This fortuitous outcome has enabled optical amplification, lasing, and wavelength conversion, functions that until recently were perceived to be beyond the reach of silicon [1-3].

Due to its centrosymmetry, bulk silicon crystal lacks second-order optical nonlinearity $\chi^{(2)}-$ the foundation of nonlinear optics. Hence, its lowest-order nonlinearity originates from the third-order susceptibility $\chi^{(3)}$, which gives rise to Raman and Kerr effects [4-5]. Raman effect, a $3^{\text {rd }}$-order nonlinearity arising from optical phonon contribution, was proposed and demonstrated in 2002 as a mean to bypass silicon's indirect bandgap limitations, and to create optical amplifiers and lasers in silicon [6]. The approach was motivated by the fact that the stimulated Raman gain coefficient in silicon is $10^{3}-$ $10^{4}$ times larger than that in silica fiber. The modal area in a silicon waveguide is roughly 100 times smaller than in fiber, resulting in a proportional increase in optical intensity. The combination makes it possible to realize chip-scale Raman devices that normally require kilometers of fiber to operate. The initial demonstration of spontaneous Raman emission from silicon waveguides in 2002 was followed by the demonstration of stimulated Raman scattering [7] and parametric Raman wavelength conversion by coherent anti-Stokes Raman scattering (CARS) [8], both in 2003. Subsequently, we witnessed the demonstration of the first silicon laser [9]. The rapid pace of progress is continuing, and the first quarter of 2005 has already seen the demonstration of direct electrical modulation of the Raman laser [10] and report of the first continuous-wave (CW) silicon Raman laser [11].

On the other hand, Kerr effect $-\mathrm{a} 3^{\text {rd }}$-order nonlinearity arising from bound electron contribution resulting in intensitydependent changes in the refractive index - in silicon is found to be more than 100 times larger in the telecommunication wavelengths than that of fused silica. Motivated by this, researchers has utilized Kerr effect in silicon to realize chipscale wavelength conversion via four-wave mixing (FWM) [12-14], parametric amplification and oscillation [15], optical switching by self-phase modulation (SPM) or cross-phase modulation (XPM) [16-17], and supercontinuum generation

Silicon Photonics and Photonic Integrated Circuits II, edited by Giancarlo Cesare Righini,

Proc. of SPIE Vol. 7719, 771904 - @ 2010 SPIE · CCC code: 0277-786X/10/\$18 · doi: 10.1117/12.858153 
[18-19]. It also leads to two-photon absorption (TPA) whenever the energy of photons exceeds the half band gap. TPA can generate free carriers and causes more severe optical loss, called free carrier absorption (FCA) - a central detrimental effect limiting the performance of virtually all nonlinear silicon photonic devices [20-21].

The question today is no longer whether silicon can exhibit nonlinear optical effects, but rather how well and how much further we can reach. To this end, instead of providing a comprehensive coverage of the numerous worldwide efforts that are advancing the state of the art, we, in this manuscript, focus on several new branches of nonlinear optical effects in silicon and the niche applications enabled these phenomena. These phenomena could provide invaluable insight into the field of nonlinear silicon photonics and open up the possibility for new classes of active silicon photonic devices. In particular, we will highlight (i) two-photon photovoltaic effect for creating energy-efficient silicon photonic devices [2225], (ii) mid-wave infrared (MWIR) silicon photonics for exploiting applications beyond telecommunication [26-32], (iii) broadband Raman effects for tailoring the Raman spectra in silicon[33-34], (iv) inverse Raman scattering for chip-scale optical signal processing [35], (v) periodically-poled silicon (PePSi) - a new technology for efficient second-order nonlinear processes in silicon [36].

\section{TWO-PHOTON PHOTOVOLTAIC (TPPV) EFFECT}

The amount of information that can be sent through an optical channel increases with optical power. Meanwhile, to address the real-estate economics of silicon wafer manufacturing, photonic devices must have footprints that are as small as possible. The concomitant reduction of a waveguide cross-section leads to an optical intensity that increases with data rate. Furthermore, in the absence of $\chi^{(2)}$, the operation of a wide range of silicon photonic devices is based on $\chi^{(3)}$, such as Kerr and Raman effects. However, high optical intensity is a prerequisite for the onset of the Kerr and Raman effects - an unfortunate situation that leads to a two-fold energy-dissipation crisis [22]. First, optical power is converted to heat through TPA. Second, photons are lost not only because of TPA, but even more so by FCA. The TPA-generated free carriers must be actively removed from the waveguide core; otherwise, they will accumulate, absorb the light and convert it into heat. While the problem can be mitigated by reducing the carrier lifetime (preventing the carriers from accumulating), processes that reduce the lifetime often result in higher optical losses.

To date, carrier sweep-out using a reverse-biased $p$ - $n$ junction diode has been the only successful approach for dealing with the problem. However, this technique has its own issues: While it sweeps out the carriers and eliminates free carrier loss, the reverse-biased diode dissipates electrical power. As a case study, as much as $1 \mathrm{~W}$ of electrical power has been consumed in enabling the operation of early continuous-wave (CW) silicon Raman lasers that generated a few milliWatts of optical power [11]. To put this into context, MOS transistors in electronics consume about a million times less power. Clearly, a more energy-efficient solution is needed.

We have previously proposed and demonstrated a new carrier sweep-out technique that not only does not require electrical power dissipation but generates electrical power as well. The technique is based on our discovery of the twophoton photovoltaic (TPPV) effect, a useful effect that allows one to sweep the carriers away and eliminate the free-
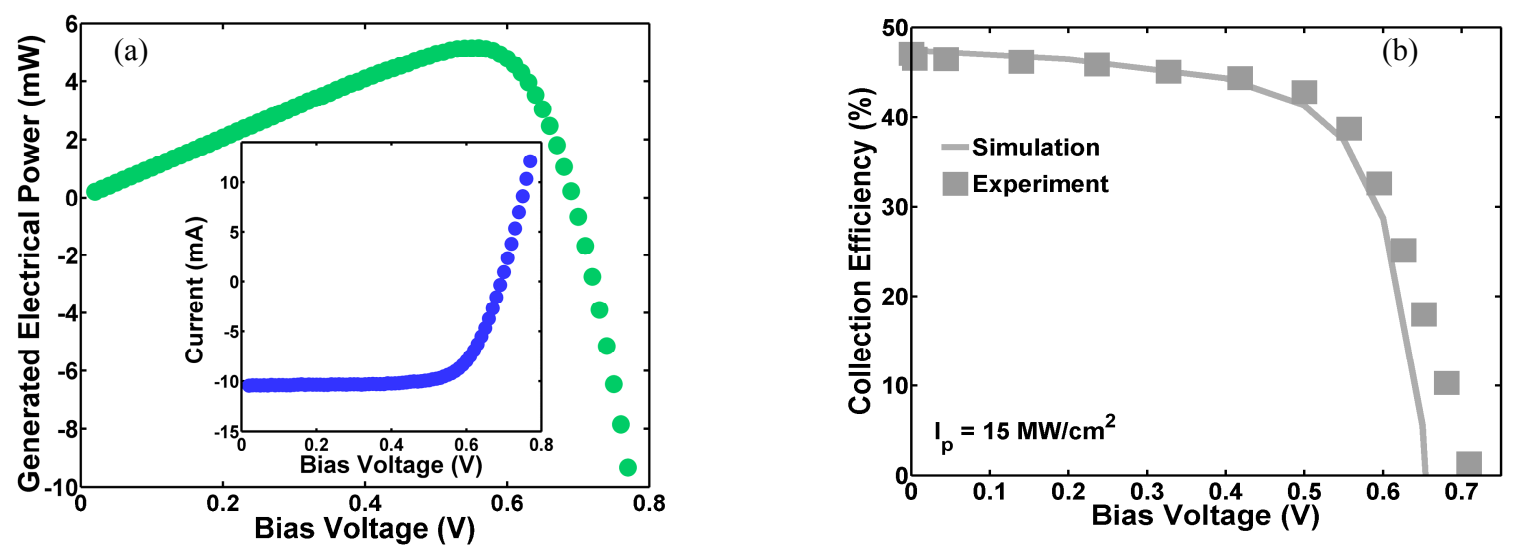

Figure 1. (a) The measured current-voltage behavior (inset) of a p-i-n diodes shows the photovoltaic effect taking place in the fourth quadrant (Current $<0$, Voltage $>0$ ) where power dissipation is negative, i.e., power generation. (b) The measured collection efficiency of two-photon photovoltaic effect at maximum power generation bias is within $39-41 \%$ compared to the theoretical maximum of $50 \%$. 
carrier absorption, while at the same time harvesting the energy of the photons lost to TPA [22-25]. The key to understanding this function is that the prerequisite for carrier sweep-out is not reverse-diode voltage but rather reverse negative current. Thus, if the $p-n$ junction is biased in the fourth quadrant of its current voltage-transfer function, the TPA-generated carriers are swept out by the internal field of the junction (created by difference in electron concentration between the $n$ and $p$ sides); yet the device has negative electrical power dissipation. In other words, it delivers power to an external load. This is because, under this operating condition, the product of the diode current and voltage and hence its power dissipation is negative (Fig. 1(a)). The sources of the generated power are the photons that were lost to TPA. This novel photovoltaic effect can be perceived as the nonlinear equivalent of the conventional photovoltaic effect used in solar cells. We have applied this energy-harvesting technique to three types of key active devices - namely, optical amplifiers [24], wavelength converters [23] and electro-optic modulators [25].

Normally, the severe TPA and FCA prevent CW operation of silicon Raman amplifiers. As a demonstration of energy harvesting in silicon optical amplifiers, we measured the Raman gain in silicon waveguides at different optical pump powers and electrical bias conditions of the diode. A CW gain of more than $6 \mathrm{~dB}$ is obtained at a reverse bias of $15 \mathrm{~V}$, but at the cost of $150 \mathrm{~mW}$ electrical power dissipation. Raman gain of about $3 \mathrm{~dB}$ is measured when the device is biased to exploit the TPPV effect, resulting in electrical power generation (negative dissipation) of around $5 \mathrm{~mW}$ (Fig. 1 and 2(a)). We have also demonstrated energy harvesting and conversion-efficiency improvements in silicon wavelength converters (Fig. 2(b)). In addition, we have proposed an electro-optic modulator that takes advantage of the TPPV effect to attain negative static power dissipation. Clearly, TPPV offers an energy-efficient solution for nonlinear silicon photonics.

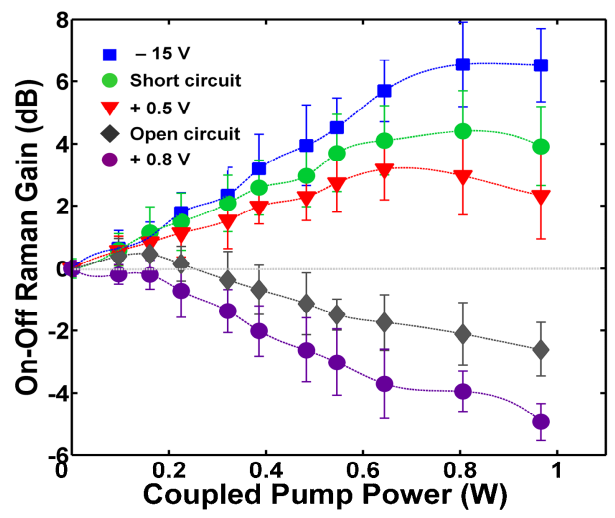

(a)

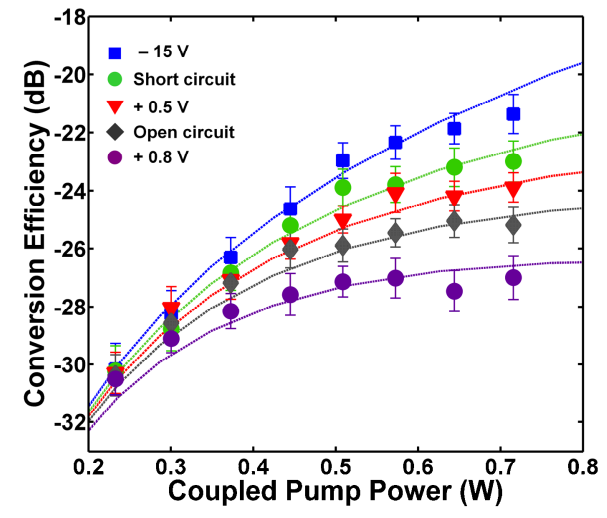

(b)

Fig. 2 (a) Measured CW Raman gain versus coupled pump power in the waveguide with high reflectivity coated facets; (b) Wavelength conversion efficiency as a function of coupled pump power under different biasing conditions in the same devices but without mirror coating. The wavelength detuning between the pump and the signal is $1 \mathrm{~nm}$.

Since the objective is not to absorb all the light, but rather to harvest the energy of the light that is absorbed by TPA, the proper definition of quantum efficiency is the number of collected carriers per absorbed photon via TPA. As two photons are required for generating one electron-hole pair, the maximum quantum efficiency is 50 percent. Indeed, we have observed good agreement between analytical and experimental results. At lower pump intensities, quantum efficiency approaches the theoretical limit of $50 \%$. The value of the efficiency at maximum power generation bias is about $40 \%$, and is nearly independent of coupled optical intensity from 5 to $150 \mathrm{MW} / \mathrm{cm}^{2}$ (Fig. 1(c)).

The TPPV offers a viable solution for achieving energy-efficient silicon photonics devices. The effect reduces the optical loss by TPA and FCA, and serendipitously converts the optical energy lost to TPA to useful electrical power. The harvested power can thus be recycled to supply electrical power to electronics on the same platform. It is even possible to create a self-powered optoelectronic chip. Beyond optical interconnects, another possible application is photovoltaic power converters (PPCs) and optically powered sensors for data links [37]. PPCs are used for remote power delivery and are optimized for a wavelength range from 1,300 to $1,550 \mathrm{~nm}$. PPCs that can provide few $\mathrm{mW}$ to $100 \mathrm{~mW}$ power are commercially available. The TPPV effect is ideal for delivering power to the sensors used for monitoring and managing fiber-optic networks. Such sensors typically measure the optical power at a given point along the fiber link. In this scenario, electrical power must be delivered to the monitoring point to provide the power needed by the photodetector and to drive the supporting electronic circuitry. With the TPPV effect, one may be able to construct a self-powered 
remote sensor that measures the optical power passing through the waveguide while at the same time providing power for the sensing circuitry. These advances will lead the way toward green integrated photonics that may have a profound effect on the telecom industry.

\section{MID-WAVE INFRARED (MWIR) SILICON PHOTONICS}

The argument of CMOS compatibility represents the traditional and still valid motivation in favor of silicon photonics. However, the case for silicon photonics is even stronger. Silicon has excellent material properties that are important in photonic devices. These include high thermal conductivity ( $\sim 10$ times higher than GaAs), high optical damage threshold ( $\sim 10$ times higher than GaAs). More importantly, the absorption spectrum of silicon, boasting a low-loss wavelength window extending from 1.1 to nearly $7 \mu \mathrm{m}$ [26] (Fig. 3(a)). We have also demonstrated, for the first time, experimentally that TPA and the TPA-generated FCA can be reduced to negligible levels by going to longer photon wavelengths for which the combined energy of two-photons is less than the band-gap of silicon [27] (Fig. 3(b)). Hence, far from being limited to the near-infrared (NIR) data communication band of $1.3-1.55 \mu \mathrm{m}$, silicon is an excellent material in the MWIR spectrum, defined loosely as the wavelength range spanning 2-6 $\mu \mathrm{m}$.

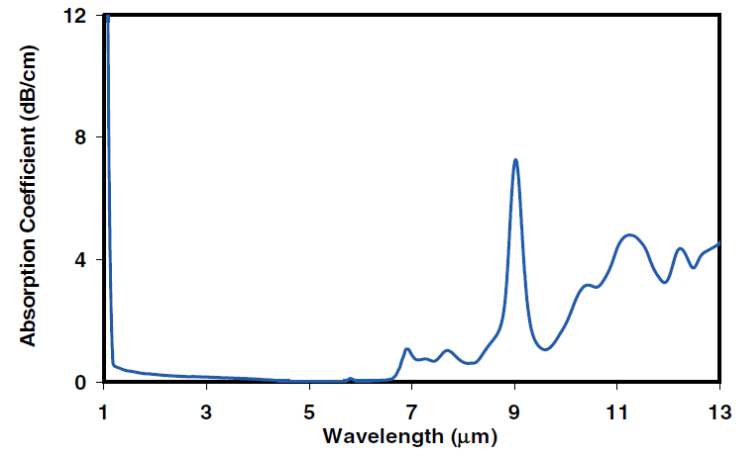

(a)

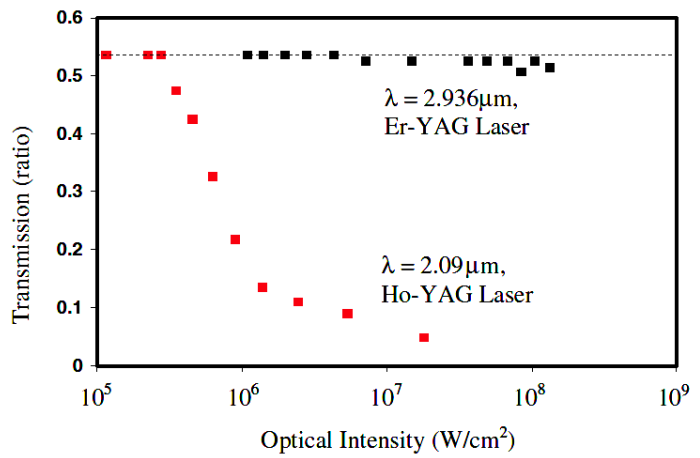

(b)

Fig. 3 (a) Linear absorption in silicon measured using an FTIR apparatus. (b) Optical transmission in silicon as a function of intensity.Two different pump sources at $2.09 \mu \mathrm{m}$ and $2.936 \mu \mathrm{m}$ were used in these experiments. The enhanced nonlinear losses at 2.09 $\mu \mathrm{m}$ due to TPA and FCA and the absence of these losses at $2.936 \mu \mathrm{m}$ are clearly seen.

MWIR laser sources and amplifiers are useful in applications such as free space communications, bio-chemical detection and certain medical procedures [38,39]. Most organic chemicals and biological agents have unique signatures in the MWIR. The strong water absorption peak at $2.9 \mu \mathrm{m}$ and the resulting applications in medicine and dentistry creates a large demand for such lasers. Typical laser sources developed for this wavelength range have not achieved wide spread use owing to the complexity, high cost of implementation and poor reliability. Moreover, there is a need for efficient and versatile amplifiers in the MWIR to be used in power-scalable master oscillator power amplifier (MOPA) systems.

Another new application is the recently proposed image pre-amplifiers [28]. Such a device employs Raman amplification combined with Talbot self imaging in a multimode waveguide. This is an effect of constructive interference among the various waveguide modes every periodic length [40]. The device consists of collinearly propagating pump and Stokes beams. The Stokes beam is amplified through energy gained from the pump beam, but at the same time, it is focused at periodic intervals along the waveguide including at the waveguide output (Fig. 4). This device has several applications, perhaps the most important of which is as an image preamplifier for laser remote sensing and imaging systems. Here, a pixilated image is simultaneously amplified and focused as it propagates through the device. By elevating the incoming signal above the thermal noise level of the optoelectronic image sensor, the preamp will improve the sensitivity of laserbased imaging systems. The technology is best suited for operation in MWIR due to the lack of TPA-induced free-carrier effects. A unique feature of the physics of Raman interactions in multimode waveguides is that the Raman amplification process is accompanied by Raman induced FWM between various spatial modes of pump and image (Stokes) beams. This complex phenomenon places a limit on the maximum achievable gain, beyond which the image begins to distort. The image may also become distorted due to preferential amplification of Stokes modes that have the highest overlap with the pump. Such effects introduce a tradeoff between the gain and image quality, however, this tradeoff can be eliminated by selectively launching the pump beam into a single higher order waveguide mode [28]. 


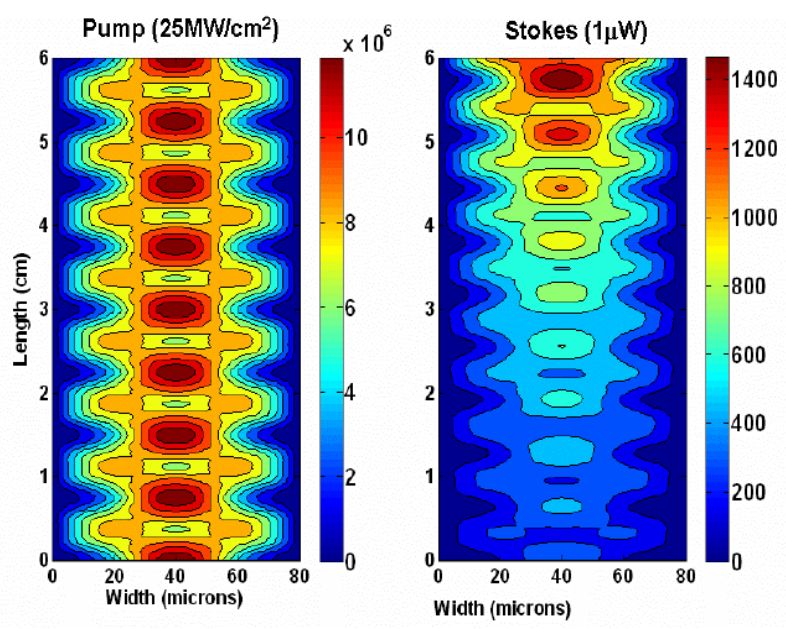

Fig. 4 Contour profile of the electric field amplitude showing the self-imaging Raman amplifier with the evolution of the pump and Stokes along the length of the multimode silicon waveguide. Pump power coupled into the waveguide is $1 \mathrm{KW}$ peak ( $1 \mathrm{~mW}$ average) and Stokes power is $1 \mu \mathrm{W}$. The air-clad waveguide width is $80 \mu \mathrm{m}$, and its thickness is $50 \mu \mathrm{m}$.

Clearly, the missing prerequisite for experimental realization of such a device is Raman amplification in the MWIR. We have reported on the first demonstration of an MWIR silicon Raman amplifier [29]. Specifically, an input signal at 3.39 $\mu \mathrm{m}$ wavelength is amplified by $12 \mathrm{~dB}$ in a $2.5 \mathrm{~cm}$ long silicon sample (see Fig. 5). The sample was pumped with $5 \mathrm{~ns}$ long pump pulses at $2.88 \mu \mathrm{m}$. It is worthwhile to point out that care must be taken to avoid other problems that are more severe in the MWIR than near-IR. Higher order nonlinear process such as three-photon absorption can occur at very high intensities. This will be highly undesirable because the $\lambda^{2}$ increase in the free carrier scattering cross section renders the presence of free carriers more problematic at MWIR than in the near-IR. In addition to absorption of light, free carrier scattering can also lead to heating of the lattice with concomitant thermal generation of additional carriers. Another difference between the two regimes is that the size of MWIR waveguides will be larger. To the extent that the carrier lifetime depends on surface recombination, the lifetime is expected to be longer in mid-IR waveguides than in near-IR waveguides. The longer lifetime enhances accumulation of any free carriers that are generated by three photon absorption, thermal generation, and defect assisted excitations.
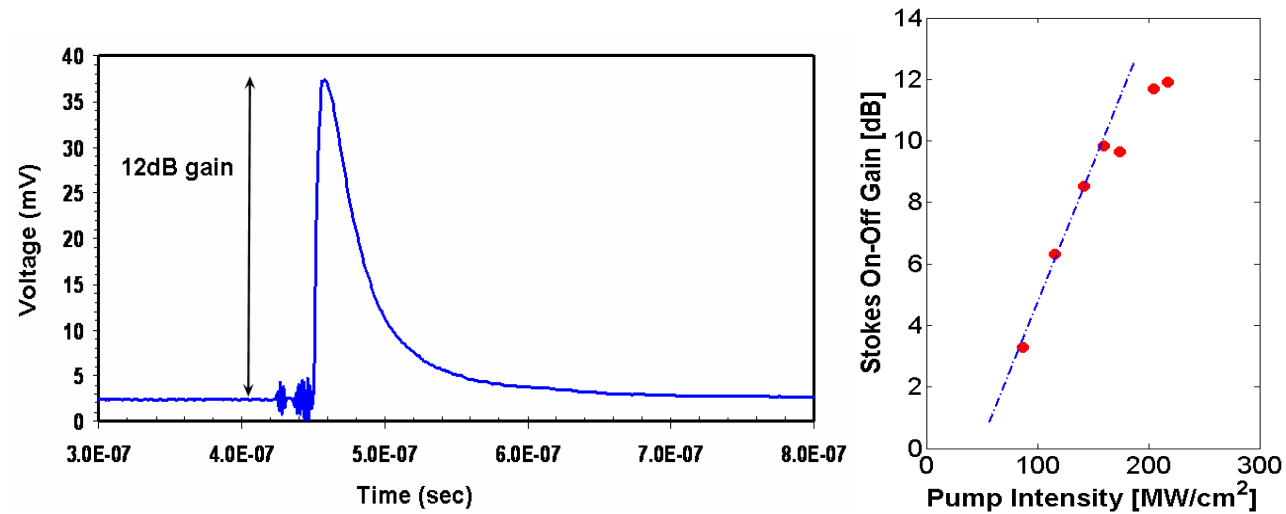

Fig. 5 (a) The time-resolved Raman amplification at $3.39 \mu \mathrm{m}$ in silicon. The typical MWIR Raman gain (on-off) obtained was $\sim 12 \mathrm{~dB}$. (b) The plot of on-off MWIR Raman gain as a function of effective pump intensity interacting with the Stokes input.

Anyhow, the absence of the TPA and its associated FCA losses, which severely limit the performance of the silicon photonic devices in the NIR, combined with unsurpassed crystal quality, high thermal conductivity, and excellent damage threshold render silicon a very attractive nonlinear optical medium at MWIR. Consequently, MWIR silicon photonics has triggered growing attention in the past 4 years [26-32]. For instance, FWM and parametric amplification at $\sim 2.2 \mu \mathrm{m}$ has recently been demonstrated in sub-micron silicon waveguides [41]. Several groups proposed different low- 
loss waveguide designs for MWIR [30-31]. By extending the utility of silicon photonics beyond data communication, we are now opening a new door to the equally important applications in biochemical sensing and laser medicine.

\section{PERIODICALLY-POLED SILICON}

Bulk silicon has a centrosymmetric crystal structure, which forbids second-order $\chi^{((2)}$ optical nonlinearity. This property limits silicon's range of applications in nonlinear optics. As a result of this fundamental limitation, nonlinear processes in silicon photonics have been based on its $\chi^{(3)}$ such as the Raman and Kerr effects. However, the disruption of crystal symmetry at the surface can enable $\chi^{(2)}$ processes such as second harmonic generation (SHG) [42, 43]. In addition, the crystal symmetry can also be perturbed by applying inhomogeneous mechanical stress (strain gradient) on the crystal [44]. Recently, strained silicon has been used to demonstrate linear electro-optic modulators [44]. The stress can be induced by depositing silicon nitride on top of waveguides, and a $\chi^{(2)}$ value of $\sim 15 \mathrm{pm} / \mathrm{V}$ has been measured with $1 \mathrm{GPa}$ stress. Motivated by these large values of $\chi^{(2)}$ we have recently proposed a new concept: periodically-poled silicon (PePSi) [36]. PePSi creates alternating stress-gradients along a silicon waveguide using a periodic arrangement of strained films. The structure creates appreciable $\chi^{(2)}$ and simultaneously achieves quasi-phase matching (QPM). Based on $\chi^{(2)}$ values reported in [44], our simulations have shown efficient MWIR generation through the quasi-phase-matched difference frequency generation process (QPM-DFG). The PePSi concept is meant to broaden the capabilities of silicon as a nonlinear optical medium.

The PePSi structure considered here is a channel waveguide integrated with two types of silicon nitride (SiN) stressed films: one with tensile stress and another with compressive stress, periodically deposited along the waveguide, as shown in Fig. 6. Hence, the sign of stress induced within the silicon core alternates along the waveguide and results in alternating dipole regions - a new type of periodically-poled structure. SiN is chosen because it achieves high film stress [45] and is compatible with CMOS processing. In addition, the stress in SiN films can be readily tailored from compressive to tensile stress depending on the deposition conditions [45]. It thus permits flexible engineering of stress, and hence $\chi^{(2)}$, in silicon. In practice, the design shown in Fig. 6 can be realized by two different SiN deposition steps, giving rise to alternating stresses along the waveguide. For MWIR applications, SOI is not the desirable platform because of the high losses of silicon dioxide at MWIR wavelengths. Instead, silicon-on-sapphire (SOS) is employed here as sapphire is transparent to MWIR.

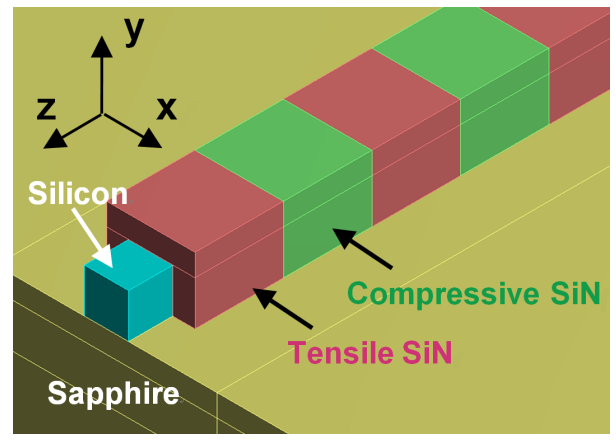

Fig. 6 An example of PePSi waveguide formed by covering a silicon channel waveguide with two types of silicon nitride (SiN) stressed films periodically along the waveguide: one induces compressive stress, whereas the other one produces tensile stress.

The PePSi waveguide we consider has the silicon core dimensions of $2 \mu \mathrm{m} \times 2 \mu \mathrm{m}$, which supports optical waveguiding in both MWIR and NIR regimes. We assume the in-plane stresses in the two different SiN films (with $1 \mu \mathrm{m}$ thickness) to be $+1 \mathrm{GPa}$ (tensile) and $-500 \mathrm{MPa}$ (compressive) [45]. The SiN stressed film period is designed to be $8 \mu \mathrm{m}$ in order to phase-match the interacting waves in the DFG process for MWIR generation (discussed later). To estimate the stressinduced $\chi^{(2)}$ in the PePSi waveguide, we simulated the stress distribution in the waveguide by ANSYS. Simulated crosssectional $\sigma_{y y}$ distributions in the tensile and compressive SiN cladding regions are shown in Fig. 7 (a) and (b), respectively. In one half of the period, when the SiN film exhibits tensile stress that deforms the waveguide, a compressive stress field is induced and confined inside the waveguide core (Fig. 7(a)) in order to counteract the deformation under elastic equilibrium. Conversely, in another half of the period, the compressive SiN cladding induces tensile stress within the silicon core (Fig. 7(b)). Also, the stress-induced $\chi^{(2)}$ distribution show a reasonably good uniformity (Fig. 7(a) - (b)). This feature is important to ensure efficient $\chi^{(2)}$ interaction of the optical modes within the 
waveguide. The picture of the periodic poling becomes more appealing when we observe the average stress $\left(\sigma_{\mathrm{yy}}\right)$ along the waveguide. As illustrated in Fig. 7(c), the periodic oscillation with $-500 \mathrm{MPa}$ peak average compressive stress and +200 MPa peak average tensile stress is evident.

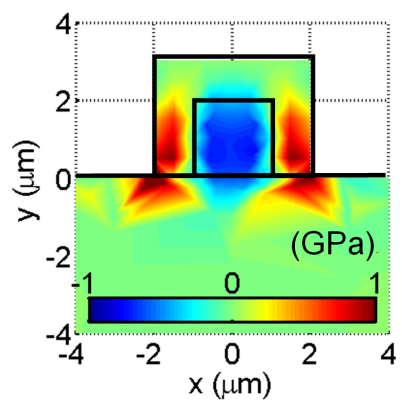

(a)

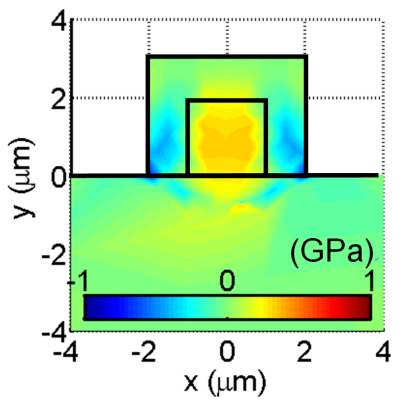

(b)

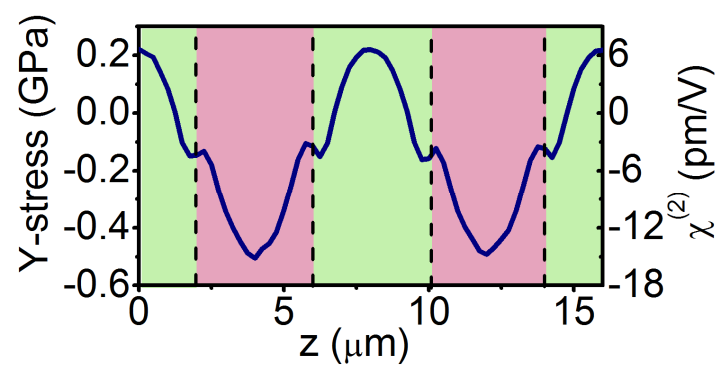

(c)

Fig. 7. Simulated cross-sectional $y$-component normal stress $\left(\sigma_{y y}\right)$ distribution where the PePSi waveguide is covered by silicon nitride films with (a) $1 \mathrm{GPa}$ (tensile) stress and (b) $-500 \mathrm{MPa}$ (compressive) stress. (c) Simulated average $\sigma_{y y}$ and estimated second order susceptibility $\left(\chi^{(2)}\right)$ induced along the waveguide (z-direction).

Based on a classical anharmonic oscillator model [46], together with the simulated stress values, the induced $\chi^{(2)}$ is estimated to have oscillatory values from $-15 \mathrm{pm} / \mathrm{V}$ to $+6 \mathrm{pm} / \mathrm{V}$ in a period of $8 \mu \mathrm{m}$ (Fig. 7(c)). This oscillatory behavior is the desired property for QPM. The estimated $\chi^{(2)}$ agrees qualitatively with the prior work for the same order of magnitude of stress [44]. It should be noted that the classical anharmonic oscillator model is meant as a heuristic tool, and should not be interpreted rigorously. A full calculation of the nonlinearity induced in silicon by applied stress remains an open question. The values measured by Jacobsen et al. [44] are the fundamental justification for the value of the nonlinear parameter used in our simulations. In fact, it was recently found that there appears to be a difference between the experimental $[43,44]$ and theoretical [42] values of $\chi^{(2)}$ reported in the literature for strained silicon, with theoretical values [42], being much lower than their experimental counterparts [43, 44]. The apparent disagreement suggests that there may be important missing physics in the existing theoretical calculations. Interestingly, the $\chi^{(2)}$ value obtained using a formula derived from a heuristic model based on the classical anharmonic oscillator [46] agrees with the measurement of Jacobson et al. In this simple model, $\chi^{(2)}$ is proportional to the total stress (integrated stress gradient) rather than the stress gradient itself. This may provide a clue regarding the present gap between theory and experiments.

The PePSi technology introduced here allows efficient silicon photonic devices based on different $\chi^{(2)}$ processes. As an example, we consider the generation of MWIR radiation in PePSi waveguide from two NIR beams using DFG. We note that MWIR generation in silicon can also be implemented by first-order stimulated Raman scattering (SRS) pumped at shorter MWIR wavelengths [29], or by cascaded SRS pumped at near infrared (NIR) wavelengths [47, 48]. In contrast, the present MWIR generation approach using PePSi is a single-step conversion from NIR via difference frequency generation (DFG). This is more favorable in terms of the wide-availability of the NIR pump sources and also more efficient in terms of circumventing the cumulative two-photon absorption (TPA) and associated free-carrier absorption (FCA) that occurs in the cascaded SRS approach.

We numerically investigated the QPM-DFG process in the PePSi waveguide using the nonlinear Schrödinger equation (NLSE), which incorporates (1) stress-induced $\chi^{(2)}$ effects, (2) $\chi^{(3)}$ effects (Kerr effect, TPA), and free-carrier effects. Taking the waveguide dispersion into account, the 8- $\mu \mathrm{m}$-period of the SiN film pattern is designed to satisfy the QPM condition in the DFG process: pump at $1.3 \mu \mathrm{m}$, signal at $1.75 \mu \mathrm{m}$ and idler at $5.1 \mu \mathrm{m}$. In the model, we input transformlimited pump and signal pulses (both with pulse width of $12 \mathrm{ps)} \mathrm{into} \mathrm{a} \mathrm{2-cm-long} \mathrm{PePSi} \mathrm{waveguide.} \mathrm{Through} \mathrm{QPM-DFG,}$ the MWIR idler pulse can be efficiently generated at $5.1 \mu \mathrm{m}$ at the waveguide output (Fig. 8 (a)). During the process, second harmonic generation (SHG) and sum frequency generation (SFG), albeit without phase matching, can generate photons with energies above the silicon bandgap. This inevitably causes single-photon absorption (SPA), which introduces additional FCA and, thus, deteriorates the DFG efficiency. We also remark that even though the idler wave suffers from larger FCA than the pump and signal waves due to the wavelength-dependence of FCA, only the trailing edge of the idler pulse is suppressed by the FCA since more free carriers are generated toward the trailing edge [49]. As a result, high peak conversion efficiency can still be obtained. As depicted in Fig. 8(b), which shows the peak conversion 
efficiency as a function of the peak pump intensity, the PePSi waveguide is able to achieve a maximum conversion efficiency of the MWIR generation by QPM-DFG as high as $\sim-3 \mathrm{~dB}(\sim 50 \%)$ under peak pump intensity of $1.5 \mathrm{GW} / \mathrm{cm}^{2}$. This intensity level is readily achievable under experimental conditions.

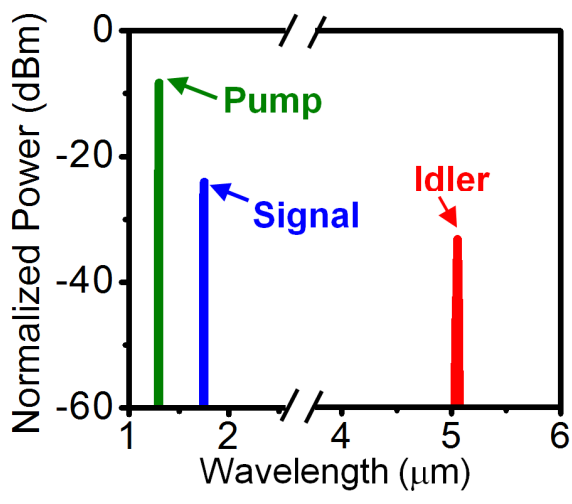

(a)

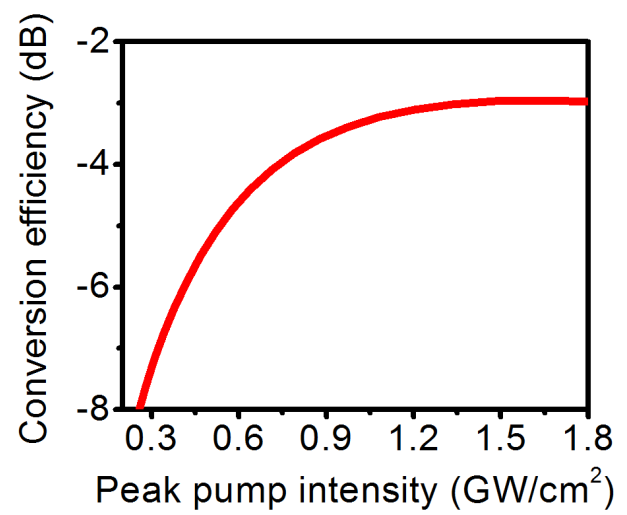

(b)

Fig. 8 (a) Calculated output spectra in a 2-cm-long PePSi waveguide (Period $\sim 8 \mu \mathrm{m}$ ): a 12-ps pump pulse at $1.3 \mu \mathrm{m}$ (input peak intensity $=1.5 \mathrm{GW} / \mathrm{cm}^{2}$ ), a 12 -ps signal pulse at $1.75 \mu \mathrm{m}$ (input peak intensity $=12.5 \mathrm{MW} / \mathrm{cm}^{2}$ ), idler at $5.1 \mu \mathrm{m}$. (b) Calculated conversion efficiency of MWIR generation.

\section{BROADBAND RAMAN EFFECTS IN SILICON}

The observation of SRS in silicon waveguides has proven that silicon is an appealing optical gain medium. Unfortunately, unless a broadband or multi-wavelength pump is used, the intrinsic $100 \mathrm{GHz}$ gain spectrum of Raman is only sufficient for amplifying two 10Gbit/s data channels spaced by the industry standard $50 \mathrm{GHz}$ optical frequency spacing. On this regard, in recent pioneering experiments, broadband parametric gain based on phase matched FWM was demonstrated in silicon waveguides $[12,15]$. In contrast to Raman gain, however, this mechanism requires phase matching, which necessitates careful control of the waveguide dimensions or application of external stress [50-52]. We recently demonstrated Raman scattering with simultaneous continuum generation in a silicon waveguide to produce broadband gain, significantly larger than the natural Raman linewidth of the medium [34]. Such achievement is realized by utilizing a pulsed pump source that is modified by SPM within the silicon medium; this nonlinear effect broadens the input pulse inside the device during propagation. Utilizing the same approach, we are also able to achieve broadband wavelength conversion via CARS [33]. Being a Raman process, broadband Raman amplification or CARS wavelength conversion is produced here without the need for any phase matching.

Fig. 9 (a) shows the measured broadband Raman gain spectra produced by 3 ps pump pulses in a 2-cm long silicon waveguide with a modal area of $\sim 2.8 \mu \mathrm{m}^{2}$. These broadened Raman spectra show rapid undulations characteristic of SPM and are shifted toward blue wavelengths due to free-carrier refraction (FCR). We point out that the natural Raman linewidth in silicon is $\sim 1 \mathrm{~nm}$. For reference, we also show the broadened pump spectra at the waveguide output for different peak power levels. The majority of the gain is shifted to wavelengths shorter than $1686 \mathrm{~nm}$, even for fairly modest pump power. At higher power, the gain spectrum develops a second lobe appearing further to the blue. Fig. 9 (b) shows the measured spectrum of broadband CARS wavelength conversion in the same silicon waveguide.

The effect of the free-carrier and Kerr nonlinearities on the pump can be qualitatively understood by considering their tandem actions. The dynamic phase shift imparted by Kerr refraction upshifts the frequency of the pulse's trailing edge and downshifts the leading edge [53]. Carrier refraction, however, upshifts both the leading and trailing slopes [49]. Thus, FCR partially negates the Kerr redshift on the leading edge but adds to the blueshift on the trailing side [49]. On the other hand, FCA preferentially attenuates the trailing half of the pulse, absorbing the new blue frequency components on this side. The net result is still a broadened, blueshifted pulse, but its spectral extent is limited to some degree by the combined nonlinearities. 


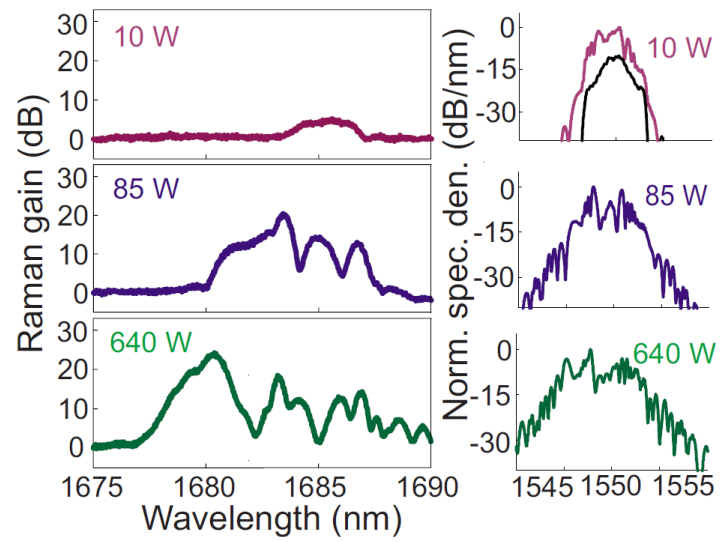

(a)

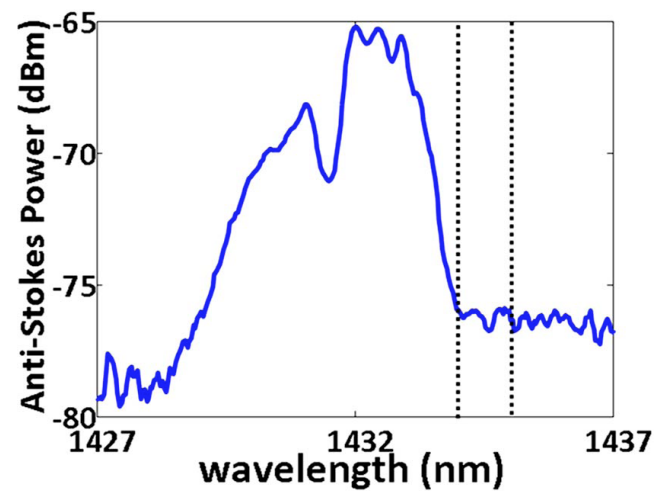

(b)

Fig. 9 (a) Measured broadband silicon Raman gain spectra with 3 ps pump pulses. (Left) Raman gain spectra produced by pump pulses with the indicated input peak power levels. (Right) Normalized spectral densities of pump pulses at the waveguide output. (b) Measured spectrum of broadband CARS conversion in silicon.

From numerical simulation based on NLSE, we also observed that the relative delay between the pump and Stokes pulses can be used to control the broadband Raman gain profile. Figure 10 shows a family of simulated gain spectra obtained by varying the initial delay between the pulses. As broadband carrier losses increase toward the trailing edge of the pump, the gain relative to the output Stokes level without the pump (on/off gain) tends to be larger on the leading edge of the pulse. We also observe that when the probe is timed on the pump's leading edge, the gain develops a multipeak structure, similar to what is observed experimentally. This impact of the relative delay may be understood by considering the evolution of the temporal profile of the pump as it propagates through the waveguide and the distribution of the frequencies generated by the SPM underneath the pump envelope (i.e., the chirp of the pump). By varying the pulse characteristics (i.e., pulse power, chirp, duration, and timing), we are able to produce a broadened gain spectrum with a tailored profile in silicon. This may be very useful for on-chip optical signal processing and producing tunable silicon lasers.
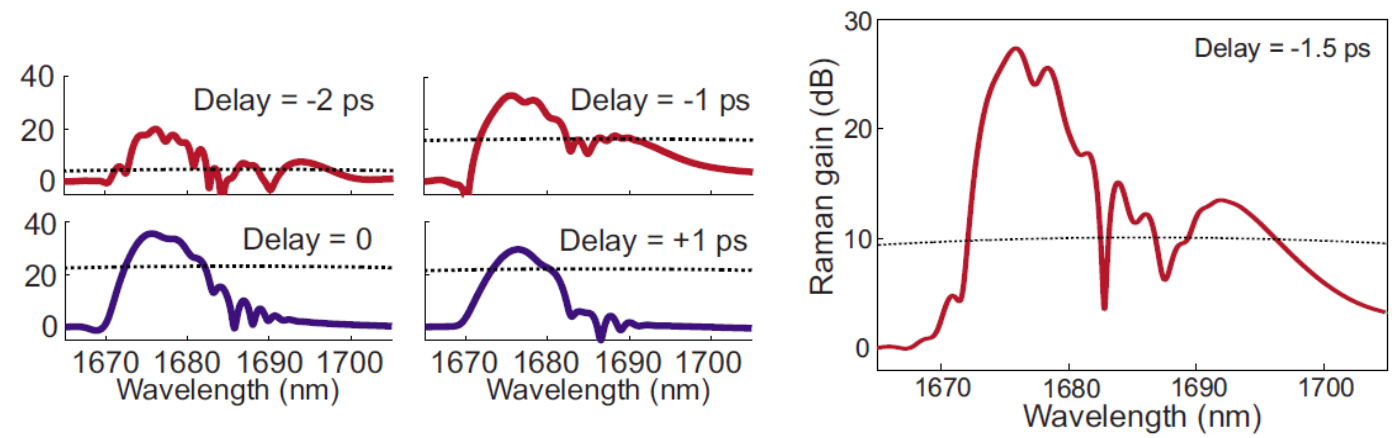

Fig. 10 Simulated Raman gain spectra at the indicated pump-probe delay (pump: 3 ps, $640 \mathrm{~W}$ ). Negative delay corresponds to probe on the leading slope of pump. Dotted lines mark "break-even" levels for the pump on/off gain. For -1.5 ps delay, the on/off gain is largest, and the spectral shape resembles that measured experimentally.

\section{INVERSE RAMAN EFFECTS IN SILICON}

Apart from the SRS effect - results in optical amplification, and the CARS effect - results in wavelength conversion, the Raman effect can also produce optical loss through inverse Raman scattering (IRS) [54,55]. In this nonlinear process, light at the anti-Stokes wavelength is attenuated in the presence of an intense pump, opposite to the exponential Stokes gain in Raman amplification. IRS was first reported by Jones and Stoicheff [54] in organic liquids and has been used as 
a technique for Raman spectroscopy [56]. Since IRS occurs at the anti-Stokes wavelength, it avoids fluorescence contamination [54,56]. Apart from its utility in spectroscopic measurement, IRS could prove to be a valuable tool for photonic signal processing. However, to the best of our knowledge, IRS has never been observed in a semiconductor medium. We recently demonstrated the resonant attenuation of an optical signal in a silicon waveguide through IRS [35]. In agreement with the known Raman characteristics of silicon, the observed attenuation bandwidth is $\sim 100 \mathrm{GHz}$, but in contrast to SRS, it is blueshifted by $15.6 \mathrm{THz}$ from the pump wavelength.

In spontaneous Raman scattering, a minute amount of Stokes light and an even smaller amount of anti-Stokes light are spontaneously radiated, with the proportion determined by the thermal occupation factor of the excited vibrational state. When Stokes or anti-Stokes input waves are added, however, the situation is dramatically different: an input Stokes wave is amplified through SRS, while an input anti-Stokes wave suffers attenuation through IRS. SRS and IRS are corollary processes, with similar spectral characteristics. Amplification of the Stokes field comes at the expense of pump, whereas, photons are transferred to the pump during the attenuation of the anti-Stokes field.

On the other hand, CARS power transfer between Stokes and anti-Stokes waves depends on the phase mismatch between the pump $\left(k_{P}\right)$, Stokes $\left(k_{S}\right)$, and anti-Stokes $\left(k_{a S}\right)$ propagation constants: $\Delta k=2 k_{P}-k_{S}-k_{a S}$. Thus, CARS can impact the attenuation suffered by an anti-Stokes signal through IRS. Even in the presence of phase mismatch, significant transfer of energy to the anti-Stokes wave can occur for high pump intensity [57]. The transfer can be reduced by eliminating Stokes input light; however, at high pump power, FWM coupled with Raman amplification can still produce substantial Stokes radiation, affecting the anti- Stokes attenuation [35].

In silicon, the landscape is complicated by additional nonlinear effects: the TPA and TPA-generated FCA. These effects result in a self-limiting process that depletes the pump intensity [49]. Simultaneously, SPM, produced by a concerted action of the Kerr nonlinearity and FCR blueshifts the pump spectrum [49]. Although FCA generally degrades the performance of silicon devices, we demonstrated that it surprisingly facilitates the observation of IRS [35]. Without FCA, the pump power remains larger throughout the waveguide, favoring the growth of the Stokes field and increasing the CARS conversion efficiency. For relatively moderate power levels, CARS contamination becomes so strong that resonant gain is observed at the anti-Stokes wavelength overwhelming the IRS induced attenuation [35]. Such "FCAenhanced effect" makes IRS in semiconductors distinctly different from that in other media.
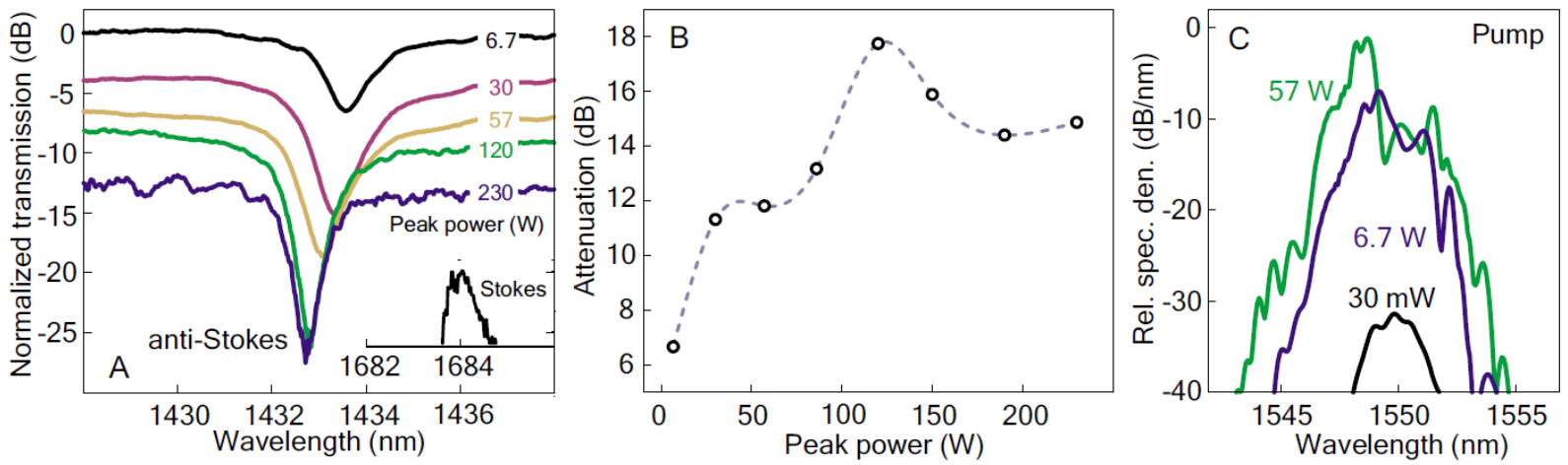

Fig. 11 Experimental observation of IRS in silicon. (a) Normalized transmission vs wavelength at indicated pump power levels. IRS causes resonant nonlinear attenuation; broadband loss arises from TPA and FCA. Inset shows transmitted Stokes signal (same $y$ axis from main plot applies). (b) Maximum resonant attenuation of anti-Stokes signal vs pump power. (c) Measured pump spectra at waveguide output. Nonlinearity blueshifts peak of pump spectrum causing blueshift in IRS line as seen in (a).

Measured IRS spectra are shown in Fig. 11(a). At the highest pump intensity, the signal minimum becomes comparable to the noise floor. Whereas IRS produces resonant attenuation, TPA (pump photon+anti-Stokes photon) and FCA produce broadband nonlinear loss. These spectral characteristics reflect the corresponding response time scales: TPA and FCA involve electronic transitions and respond essentially instantaneously (although FCA requires nanoseconds to subside); IRS, being a Raman effect, responds according to the phonon ring down time, which occurs on a picosecond time scale. The resonant IRS attenuation (discounting the broadband absorption) increases rapidly with pump power initially, as expected, but levels off and decreases slightly for high power (Fig. 11(b)). Nevertheless, IRS attenuation values $\sim 15 \mathrm{~dB}$ are readily observed. At high pump intensities, we observe the generation of Stokes signal (see Fig. 11(a), inset). The generation of this signal arises primarily from Raman amplification of power transferred coherently from the 
anti-Stokes probe to the Stokes wavelength. This Stokes signal is also transferred back to the anti-Stokes wavelength via CARS, which tends to limit the observable IRS attenuation. This process coupled with the self-limiting nature of the pump power are likely responsible for the high-power reduction in IRS absorption illustrated in Fig. 11(b). It may also be noted that the IRS spectrum shifts toward shorter wavelengths as the pump intensity is increased, which results from the SPM-induced blueshift of the pump spectral peak (Fig. 11(c)).

\section{CONCLUSION}

Not meant as a comprehensive review, this manuscript aims to provide a new perspective of nonlinear silicon photonics by introducing some new branches in this field, which could open up the possibility for new classes of active silicon photonic devices. In summary, adding TPPV effect to silicon photonic devices may allow us to harvest the optical loss to electrical power and simultaneously to mitigate the TPA and the associated FCA optical loss. Such advance will lead the way toward green integrated photonics. The absence of the nonlinear losses in MWIR combined with unsurpassed crystal quality, high thermal conductivity and excellent optical damage threshold render silicon a very attractive nonlinear optical medium. Exploiting these facts, MWIR silicon photonics can extend the utility of silicon photonics beyond telecommunication into applications in medicine, biochemical sensing, laser imaging detection and ranging (LIDAR). PePSi offers a path to realize efficient wave mixing devices based on $\chi^{(2)}$, such as efficient MWIR generation based on DFG, and possibly terahertz $(\mathrm{THz})$ generation as silicon exhibits excellent transmission properties at $\mathrm{THz}$ frequencies. When combined with piezoelectric stressed layers, this technology can also offer the capability to dynamically control the $\chi^{(2)}$ in silicon [51-52]. The ability to produce a broadened gain spectrum with a tailored profile in silicon may be very useful for on-chip optical signal processing and producing tunable silicon lasers. Last but not least, IRS may be very useful for optical signal processing on silicon chips and is an important complementary tool to Raman gain and wavelength conversion via SRS and CARS.

Finally, research into nonlinear optical interactions is leading to unexpected scientific discoveries. As a case in point, we have recently reported that fluctuations of Raman amplified pulses, in the presence of a noisy pump, follow extremevalue statistics - highly non-Gaussian distributions that have been surprisingly successful in describing the frequency of occurrence of extreme events ranging from stock market crashes and natural disasters, the structure of biological systems and fractals, and optical rogue waves [58,59]. These distributions predict that events much larger than the mean can occur with significant probability, in stark contrast to the ubiquitous Gaussian distribution which heavily favors events close to the mean. Experimentally, we have found that $16 \%$ of the amplified pulses account for $84 \%$ of the pump energy transfer, an uncanny resemblance to the empirical 80/20 rule that describes important observation in socioeconomics. A simple mathematical model provides insight into this fascinating behavior and how it emerges in such diverse and seemingly unrelated fields of physical and social sciences.

\section{ACKNOWLEDGEMENT}

Much of the progress described in this manuscript would not have been possible without the generous support from the Defense Advanced Research Project Agency (DARPA). We are particularly indebted to Dr. Jag Shah of DARPA for his visionary support of silicon photonics.

\section{REFERENCES}

[1] L. Pavesi and G. Guillot, Optical interconnects: the Silicon approach (Springer, 2006).

[2] G. T. Reed and A. P. Knights, Silicon Photonics: An Introduction, Chichester, U.K. (Wiley 2004).

[3] B. Jalali and S. Fathpour, "Silicon Photonics," IEEE J. Lightwave Technol. 24, 4600-4615 (2006).

[4] Q. Lin, Oskar J. Painter, and Govind P. Agrawal, "Nonlinear optical phenomena in silicon waveguides: modeling and applications," Opt. Express 15, 16604-16644 (2007).

[5] B. Jalali, V. Raghunathan, D. Dimitropoulos, O. Boyraz, "Raman-based silicon photonics" IEEE J. Sel. Top. in Quant. Electron. 12, 412 - 421 (2006).

[6] R. Claps, D. Dimitropoulos, Y. Han, B. Jalali, "Observation of Raman emission in silicon waveguides at 1.54 $\mu \mathrm{m}$,’Opt. Exp. 10, 1305-1313 (2002). 
[7] R. Claps, D. Dimitropoulos, V. Raghunathan, Y. Han, B. Jalali, "Observation of stimulated Raman scattering in Silicon waveguides", Opt. Exp. 11, 1731-1739 (2003).

[8] R. Claps, V. Raghunathan, D. Dimitropoulos, B. Jalali, “Anti-stokes Raman conversion in silicon waveguides", Opt. Exp. 11, 2862-2872 (2003).

[9] O. Boyraz and B. Jalali, "Demonstration of a silicon Raman laser," Opt. Express, 12, 5269-5273 (2004).

[10] O. Boyraz and B. Jalali, "Demonstration of directly modulated silicon Raman laser," Opt. Express, 13, 796800 (2005).

[11]H. Rong, R. Jones, A. Liu, O. Cohen, D. Hak, A. Fang, and M. Pannicia, "A continuous-wave Raman silicon laser," Nature, 433, 725-728 (2005).

[12] M. A. Foster, A. C. Turner, R. Salem, M. Lipson, and A. L. Gaeta, "Broad-band continuous-wave parametric wavelength conversion in silicon nanowaveguides," Opt. Express 15, 12949-12958 (2007).

[13] R. L. Espinola, J. I. Dadap, R. M. Osgood, Jr., S. J. McNab, and Y. A. Vlasov, "C-band wavelength conversion in silicon photonic wire waveguides," Opt. Express 13, 4341-4349 (2005).

[14] H. Fukuda, K. Yamada, T. Shoji, M. Takahashi, T. Tsuchizawa, T. Watanabe, J. Takahashi, and S. Itabashi, "Four-wave mixing in silicon wire waveguides," Opt. Express 13, 4629-4637 (2005).

[15] M. A. Foster, A. C. Turner, J. E. Sharping, B. S. Schmidt, M. Lipson, and A. L. Gaeta, "Broad-band optical parametric gain on a silicon photonic chip," Nature 441, 960-963 (2006).

[16] O. Boyraz, T. Indukuri, and B. Jalali, "Self-phase-modulation induced spectral broadening in silicon waveguides," Opt. Express 12, 829-834 (2004).

[17]E. Dulkeith, Y. A. Vlasov, X. Chen, N. C. Panoiu, and R. M. Osgood, Jr., "Self-phase-modulation in submicron silicon-on-insulator photonic wires," Opt. Express 14, 5524-5534 (2006).

[18] O. Boyraz, P. Koonath, V. Raghunathan, and B. Jalali, "All optical switching and continuum generation in silicon waveguides," Opt. Express 12, 4094-4102 (2004).

[19] I-W. Hsieh, X. Chen, X. Liu, J. I. Dadap, N. C. Panoiu, C. Chou, F. Xia, W. M. Green, Y. A. Vlasov, and R. M. Osgood, "Supercontinuum generation in silicon photonic wires," Opt. Express 15, 15242-15249 (2007).

[20] T. K. Liang and H. K. Tsang, "Role of free carriers from two-photon absorption in Raman amplification in silicon-on-insulator waveguides," Appl. Phys. Lett. 84, 2745-2747 (2004).

[21]R. Claps, V. Raghunathan, D. Dimitropoulos, and B. Jalali, "Influence of nonlinear absorption on Raman amplification in silicon waveguides," Opt. Express 12, 2774-2780 (2004).

[22] S. Fathpour, K.K. Tsia, and B. Jalali, "Two-photon photovoltaic effect in silicon," IEEE Journal of Quantum Electronics, 43, 1211-1217, (2007).

[23] K. K. Tsia, S. Fathpour, and B. Jalali, "Energy harvesting in silicon wavelength converters," Opt. Express 14, 12327-12333 (2006).

[24] S. Fathpour, K. K. Tsia, and B. Jalali, "Energy harvesting in silicon Raman amplifiers," Applied Physics Letters, 89, pp. 061109 1-3, (2006).

[25] S. Fathpour and B. Jalali, "Energy harvesting in silicon optical modulators," Opt. Express 14, 10795-10799 (2006)

[26] B. Jalali, V. Raghunathan, R. Shori, S. Fathpour, D. Dimitropoulos, and O. Stafsudd, "Prospects for silicon midIR Raman lasers," IEEE J. Sel. Top. Quantum Electron. 12, 1618-1627 (2006).

[27] V. Raghunathan, R. Shori, O. M. Stafsudd, and B. Jalali, "Nonlinear absorption in silicon and the prospects of mid-infrared Silicon Raman laser," Phys. Status Solidi. (A) 203, R38-R40 (2006).

[28] V. Raghunathan, H. Renner, R. R. Rice, and B. Jalali, "Self-imaging silicon Raman amplifier," Opt. Express 15, 3396-3408 (2007).

[29] V. Raghunathan, D. Borlaug, R. R. Rice, and B. Jalali, "Demonstration of a Mid-infrared silicon Raman amplifier," Opt. Express 15, 14355-14362 (2007).

[30] M. M. Milošević, P. S. Matavulj, P. Y. Yang, A. Bagolini, and G. Z. Mashanovich, "Rib waveguides for midinfrared silicon photonics," J. Opt. Soc. Am. B 26, 1760-1766 (2009).

[31] S. Y. Cho and R. A. Soref, "Low-loss silicide/silicon plasmonic ribbon waveguides for mid- and far-infrared applications," Opt. Lett. 34, 1759-1761 (2009).

[32]R. A Soref, S. J. Emelett, W. R. Buchwald, "Silicon waveguided components for the long-wave infrared region," J. Opt. A: Pure Appl. Opt. 8 840-848 (2006).

[33] P. Koonath, D. R. Solli, and B. Jalali, "Broadband coherent anti-Stokes Raman scattering in silicon," Opt. Lett. 35, 351-353 (2010).

[34]D. R. Solli , P. Koonath, and B. Jalali, "Broadband Raman amplification in silicon," Appl. Phys. Lett. 93, 
191105 (2008)

[35] D. R. Solli, P. Koonath, and B. Jalali, "Inverse Raman scattering in silicon: A free-carrier enhanced effect, "Phys. Rev. A 79, 053853 (2009)

[36] N. K. Hon, K. K. Tsia, D. R. Solli, and B. Jalali, "Periodically-Poled Silicon", Appl. Phys. Lett. 94, 091116 (2009).

[37] J-G. Werthen, S. Widjaja, T-C. Wu and J. Liu "Power over Fiber: A Review ofReplacing Copper by Fiber in Critical Applications” Proc. of SPIE 5871, 58710C.1-58710C.6 (2005).

[38]I. T. Sorokina and K. L. Vodpyanov, Solid state mid infrared laser sources, (Springer Topics in AppliedPhysics, 2003).

[39] A. Kier, ed., Mid infrared semiconductor optoelectronics, (Springer series in Optoelectronics, 2006).

[40]L. B. Soldano and E. C. M. Pennings, "Optical multi-mode interference devices based on selfimaging:Principles and Applications,” J. Lightwave Technol. 13, 615-627 (1995).

[41] X. Liu, R. M. Osgood, Jr., Y. A. Vlasov \& W. M. J. Green, "Mid-infrared optical parametric amplifier using silicon nanophotonic waveguides," arXiv:1001.1533v1 (2010).

[42] J. Y. Huang, "Probing Inhomogeneous Lattice Deformation at Interface of Si(111)/SiO2 by Optical SecondHarmonic Reflection and Raman Spectroscopy," Jpn. J. Appl. Phys. 33, 3378 (1994).

[43] S. V. Govorkov, V. I. Emel'yanov, N. I. Koroteev, G. I. Petrov, I. L. Shumay, V. V. Yakovlev, and R. V. Khokhlov, "Inhomogeneous deformation of silicon surface layers probed by second-harmonic generation in reflection," J. Opt. Soc. Am. B 6, 1117-1124 (1989).

[44] R. S. Jacobsen, K. N. Andersen, P. I. Borel, J. Fage-Pedersen, L. H. Frandsen, O. Hansen, M. Kristensen, A. V. Lavrinenko, G. Moulin, H. Ou1, C. Peucheret, B. Zsigri, A. Bjarklev, W. Wu, J. Sun, K. Cao, S. Wang, and B. Shi, "Strained silicon as a new electro-optic material, "Nature 441, 199-202 (2006).

[45] P. Temple-Boyer, C. Rossi, E. Saint-Etienne, and E. Scheid, "Residual stress in low pressure chemical vapor deposition SiNx films deposited from silane and ammonia,” J. Vac. Sci. Technol. A 16, 2003 (1998).

[46] R. W. Boyd, Nonlinear Optics (Academic Press, New York, 1994).

[47]H. Rong, S. Xu, O. Cohen, O. Raday, M. Lee, V. Sih \& M. Paniccia, “A cascaded silicon Raman laser,” Nature Photonics 2, 170 (2008).

[48] M. Krause, R. Draheim, H. Renner and E. Brinkmeyer, "Cascaded silicon Raman lasers as mid-infrared sources,"Electron. Lett. 42, 1224 (2006).

[49] P. Koonath, D. R. Solli, and B. Jalali, "Limiting nature of continuum generation in silicon," Appl. Phys. Lett. 93, 091114 (2008).

[50] V. Raghunathan, and B. Jalali, "Stress-induced phase matching in silicon waveguides," Conference of Lasers and Electro-Optics (CLEO), Long Beach CA (2006) Paper CMK5.

[51] K. K. Tsia, S. Fathpour, and B. Jalali, "Electrical control of parametric processes in silicon waveguides," Opt. Express 16, 9838-9843 (2008).

[52]K. K. Tsia, S. Fathpour, and B. Jalali, "Electrical tuning of birefringence in silicon waveguides," Appl. Phys. Lett. 92, 061109 (2008).

[53] G. P. Agrawal, Nonlinear Fiber Optics, 4th ed. Academic, San Diego (2007).

[54] W. J. Jones and B. P. Stoicheff, “Inverse Raman spectra: induced absorption at optical frequencies,"Phys. Rev. Lett. 13, 657 (1964).

[55] A. D. Buckingham, “Theory of the Stimulated Raman and Related Effects,"J. Chem. Phys. 43, 25 (1965).

[56] W. Kiefer and D. A. Long (Ed.), Non-linear Raman Spectroscopy and Its Chemical Applications, Bad Winsheim, Germany, (1982).

[57] P. V. Koonath, D. R. Solli, and B. Jalali, "High Efficiency CARS Conversion in Silicon," in Conference on Lasers and Electro-Optics/Quantum Electronics and Laser Science Conference and Photonic Applications Systems Technologies, paper CThE3 (2008).

[58] D. Borlaug and B. Jalali, "Extreme-value statistics in silicon photonics," Lasers and Electro-Optics Society (LEOS) annual meeting, Newport Beach, CA, invited paper (2008).

[59] D. R. Solli, C. Ropers, P. Koonath, and B. Jalali, "Optical rogue waves," Nature 450, 1054 (2007). 